\title{
Determinación de un modelo de cinética de cristalización de $\mathrm{ZnO}$
}

\author{
Ana C. Alanís ${ }^{\mathrm{a}}$, Graciela Maawad ${ }^{\mathrm{a}}$, Carolina Platt ${ }^{\mathrm{a}}$, Franz W. Voss ${ }^{\mathrm{a}}$, Alejandro J. Alvarez ${ }^{\mathrm{a} *}$ \\ ${ }^{a}$ Instituto Tecnológico y de Estudios Superiores de Monterrey (ITESM), Campus Monterrey, Ave. Eugenio Garza Sada 2501 Sur, Colonia \\ Tecnológico. C.P. 64849, Monterrey, Nuevo León, México. \\ *E-mail alejandro.alvarez@itesm.mx
}

Recibido 02-septiembre-2013, Aceptado 25-septiembre-2013

\section{Resumen}

En la actualidad, los estudios sobre el mecanismo de cristalización, en particular el fenómeno de la nucleación, son muy limitados. Conocer las tasas de crecimiento de los cristales es de suma importancia si se desea sintetizar cualquier sólido cristalino y es de especial interés si se buscan un tamaño y una geometría de partícula específicos. En el caso particular de la síntesis de nanopartículas de óxido de zinc, conocer el mecanismo de cristalización y tener un modelo de la cinética de la misma resulta de interés para conocer las condiciones a las que el nacimiento de cristales se favorece. Tres series de experimentos se llevaron a cabo con tres tiempos de residencia distintos para alterar la sobresaturación del sistema y conocer el efecto de ésta sobre la nucleación y el crecimiento de los cristales de ZnO. El modelo encontrado, con las constantes de nucleación y de crecimiento, describe una relación lineal entra la sobresaturación y la tasa de crecimiento de cristales de $\mathrm{ZnO}$ y puede ser de utilidad para la síntesis del mismo.

\section{Introducción}

El propósito de la presente investigación es la modelación matemática de la cinética de cristalización del óxido de zinc (con fórmula $\mathrm{ZnO}$ ) en el marco de un proyecto de investigación de mayor alcance, llevado a cabo por el M.C. Augusto Gerling del ITESM, cuya finalidad es sintetizar cristales de $\mathrm{ZnO}$ con las características morfológicas adecuadas para filtrar ciertas longitudes de onda de radiación electromagnética. Dicho modelo matemático fue generado a través del método del balance poblacional descrito por Berglund [1], a partir de datos discretos de la distribución del tamaño de cristales. De éstos se obtuvieron las constantes de nucleación y crecimiento, $k_{b}$ y $k_{g}$, respectivamente, así como los órdenes de ambos fenómenos de cristalización, $b$ y $g$, para obtener la tasa de nucleación $B^{o}$ con la ecuación

$\mathrm{B}^{o}=k_{b}\left(C-C_{s a t}\right)^{b}$

y la tasa de crecimiento $G$ con la ecuación

$\mathrm{G}=k_{g}\left(C-C_{\text {sat }}\right)^{g}$

en función de la sobresaturación de una solución de óxido de zinc, $C-C_{\text {sat }}$. Esta última variable se define como la diferencia entre la concentración de la solución y la de saturación a la misma temperatura.

Para la obtención de los datos a analizar se construyó un sistema continuo de cristalización del tipo suspensión mezclada, remoción de producto mezclado (MSMPR, por sus siglas en inglés) [1] a temperatura y concentración total controladas, variando el tiempo de residencia y determinando concentraciones de salida y distribución del tamaño de cristal.

\section{Óxido de Zinc}

El óxido de zinc es un compuesto inorgánico de color blanco, cuyas partículas tienen la capacidad de bloquear rayos UVA (320-400 $\mathrm{nm})$ y UVB $(280-320 \mathrm{~nm})$, por lo que actualmente se utiliza en la formulación de bloqueadores solares comerciales.

La morfología y tamaño del cristal son de suma importancia para bloquear los rayos UV y varían de acuerdo al método de cristalización que se usa. Los principales factores que afectan la morfología y tamaño de las nanopartículas son: $\mathrm{pH}$, temperatura, tiempo de cristalización, tipo de solventes y reactivos usados, así como su concentración. Por otro lado, las principales estructuras cristalinas del $\mathrm{ZnO}$ son la Rocksalt, Zinc Blende y Wurtzite, siendo esta última la que favorece el bloqueo de rayos UV.

\section{Cristalización}

El proceso de cristalización consiste en la formación de partículas sólidas a partir de una fase homogénea, generalmente líquida. Durante el proceso de formación de un cristal, las moléculas tienden a fijarse sobre un cristal preexistente compuesto por el mismo tipo de moléculas, debido a que encajan mejor en el enrejado cristalino.

La cristalización una técnica de separación sólidolíquido, basada en la diferencia de solubilidad de la sustancia en un solvente a distintas temperaturas. Para facilitar la recuperación del material inicial, es conveniente que el compuesto sea altamente soluble a altas temperaturas y poco soluble a bajas. El óxido de zinc presenta este comportamiento en solución acuosa de cloruro de amonio como se muestra en la Tabla 1 
Tabla 1. Datos de solubilidad del $\mathrm{ZnO}$ en cloruro de amonio

\begin{tabular}{|c|c|c|c|c|c|c|}
\hline \multicolumn{7}{|c|}{$\begin{array}{l}\text { Solubilidad del óxido de zinc en solución de cloruro de } \\
\text { amonio }\end{array}$} \\
\hline \multirow{2}{*}{$\begin{array}{c}\text { Composición } \\
\text { del solvente } \\
\left(\mathrm{g} \mathrm{NH}_{4} \mathrm{Cl}\right. \\
\left./ 100 \mathrm{ml} \mathrm{H}_{2} \mathrm{O}\right)\end{array}$} & \multicolumn{6}{|c|}{ Solubilidad $=\mathrm{g} \mathrm{ZnO} / 100 \mathrm{ml} \mathrm{H}_{2} \mathrm{O}$} \\
\hline & $20^{\circ} \mathrm{C}$ & $40^{\circ} \mathrm{C}$ & $60^{\circ} \mathrm{C}$ & $7^{\circ} \mathrm{C}$ & $80^{\circ} \mathrm{C}$ & $90^{\circ} \mathrm{C}$ \\
\hline 10 & 0.4 & 0.7 & 1.3 & --- & 1.8 & --- \\
\hline 15 & 0.8 & 1.6 & 2.6 & --- & 3.5 & --- \\
\hline 20 & 0.8 & 1.6 & 4.6 & --- & 7.5 & --- \\
\hline 25 & 1.2 & 2.5 & 6.0 & 10.2 & 11.0 & 11.6 \\
\hline 30 & 1 & 2.3 & 5.0 & 8.4 & 13.2 & 14.6 \\
\hline
\end{tabular}

El proceso de formación de cristales consiste en una etapa inicial de nucleación durante la cual las moléculas de soluto comienzan a moverse en el solvente para formar agrupaciones que tienen un periodo de vida corto, denominados clusters. Posteriormente algunos de los clusters, crecen hasta alcanzar una dimensión macroscópica, mientras que otros se fragmentan.

De acuerdo a Söhnel et al., la nucleación se define como "[...] la formación de agrupamientos de átomos, moléculas o iones constituyentes de la nueva fase en pequeñas zonas separadas en el interior de la antigua fase" [2]. La tasa de nucleación es la rapidez del cambio de fase que depende del número de partículas que se forman por unidad de volumen del sistema en el que ocurre la nucleación, por unidad de tiempo. Ésta depende del grado de sobresaturación en el sistema, ya que, si es bajo, la rapidez por lo general será menor. [2] Además de la nucleación, existen otros fenómenos de cristalización, tales como el crecimiento, la aglomeración y el desgaste [3, p. 1], siendo el crecimiento el más importante. Este fenómeno implica la adición de partículas antes disueltas en el sistema a la superficie de cristales ya existentes. La distribución del tamaño de cristal, su tamaño medio, su pureza y su morfología están fuertemente influenciadas por tres factores controlables: la geometría y el tamaño del cristalizador; las condiciones de operación, principalmente temperatura y tiempo de residencia; y las propiedades de las fases sólida y líquida. [3]

Una manera de estimar el número y tamaño de los cristales es en función de las tasas a las cuales suceden la nucleación y el crecimiento. Si la tasa de nucleación es alta, los cristales serán pequeños y habrá mayor cantidad de ellos. Por el contrario, si la velocidad de crecimiento es elevada, comparada con la de nucleación, los cristales serán menores en cantidad pero de mayores dimensiones. Asimismo, un enfriamiento rápido y la subsecuente sobresaturación producen, por lo general, cristales más pequeños, mientras que uno lento produce cristales más grandes.

La cuantificación de la etapa de nucleación puede llegar a ser inexacta como menciona Skoog, et al., quien comenta que "[...] se han hecho múltiples intentos para expresar cuantitativamente la variación de la tasa de nucleación en función de la sobresaturación en casos típicos. Las dificultades experimentales asociadas con las mediciones necesarias han resultado ser muy grandes, de modo que hasta el presente no se ha obtenido una solución inequívoca del problema" [4].

Por otro lado, el crecimiento de las partículas se puede describir experimentalmente con mayor facilidad. Por ello, este proceso se ha estudiado con mayor detalle que la nucleación. En esta etapa los cristales comienzan a aumentar de tamaño y el comportamiento gráfico de la tasa de crecimiento en función de la sobresaturación resulta ser aproximadamente una línea recta.

Tomando en cuenta lo anterior, el control de las etapas de nucleación y crecimiento durante la cristalización tiene el potencial de producir partículas bien definidas con estructuras únicas en rangos de nanómetros y micrómetros. El grado de sobresaturación dictaminará la etapa controlante y ésta se puede manipular modificando la solubilidad del precipitado o su concentración. Las variaciones de temperatura o de la composición del disolvente alteran la solubilidad. Las modificaciones de la concentración de los reactivos o de la velocidad con la que se mezclan afectan a la concentración del soluto en la solución.

\section{Cristalizador MSMPR}

Un cristalizador de Suspensión Mezclada y Remoción de Producto Mezclado (MSMPR) puede entenderse fácilmente como un cristalizador dentro del cual aplican las mismas suposiciones que para un reactor tipo CSTR. La primera suposición es la de mezclado perfecto. De ella derivan que la concentración de la solución, la concentración de partículas suspendidas, la temperatura y la distribución de tamaño de cristal se consideren constantes en todo el sistema y que dichos parámetros también sean iguales en la salida del sistema. Se supone también que al sistema no entran cristales suspendidos, sino una solución homogénea sin sobresaturación. Se considera que la nucleación es el único fenómeno influyente en la formación de nuevos cristales, despreciando así los fenómenos de fragmentación y desgaste. De manera análoga, se desprecia la aglomeración, teniendo así el crecimiento como único fenómeno significativo para el aumento de tamaño de los cristales. Se considera también que el factor de forma $k_{v}$ no es función del tamaño de los cristales. Esta última suposición permite describir los diversos rangos de tamaño de cristal con una sola longitud característica L. [1]

\section{Balance Poblacional}

El análisis de la distribución de tamaño de cristal se beneficia de una herramienta matemática llamada 'balance poblacional' que se asemeja a los balances de materia y energía, tan utilizados en el análisis de procesos. La premisa sobre la cual se construye esta teoría dicta que el cambio en el número de cristales pertenecientes a un rango de tamaños es igual a la cantidad de cristales que, gracias al crecimiento, 


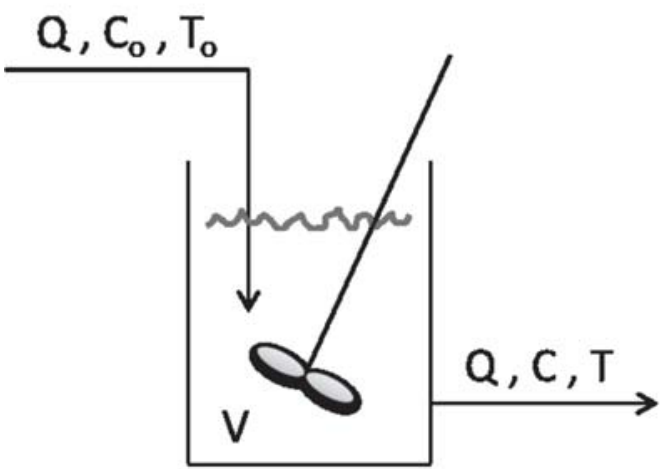

Figura 1. Diagrama de un MSMPR

superan el límite inferior de dicho rango menos aquéllos que sobrepasan el superior por el mismo motivo.

La forma común de registrar el número de cristales es utilizando una distribución acumulativa $N(L)$, en la que se cuenta el número de cristales en un volumen determinado y la cual tiene unidades típicas de número/volumen. Los datos registrados como número de cristales por unidad de volumen pueden ser transformados para obtener una distribución de densidad $n(L)$, que relaciona a la vez el número de cristales con su tamaño y el volumen del sistema. Esta última tiene unidades de número/(tamaño*volumen). Esta transformación se entiende con la ecuación

$$
\mathrm{n}=\left(\frac{d N}{d L}\right)
$$

De igual manera los datos de densidad poblacional pueden ser transformados en datos de número de cristales mediante la ecuación

$$
\mathrm{N}(\mathrm{L})=\int_{0}^{L} n(L) d L
$$

Manejar los datos experimentales transformados a densidad poblacional resulta muy útil, sin embargo, no es posible medir directamente datos de esta índole. Otra ventaja de la distribución de densidad es la posibilidad de encontrar el número total de cristales por unidad de volumen $N_{T}$ mediante la ecuación

$$
\mathrm{N}_{\mathrm{T}}(\mathrm{L})=\int_{0}^{\infty} n(L) d L
$$

así como la masa total por unidad de volumen $M_{T}$ con la ecuación

$$
\mathrm{M}_{\mathrm{T}}=\rho_{v} \int_{0}^{\infty} L^{3} n(L) d L
$$

donde $\rho$ es la densidad del sólido y $k_{v}$ el factor volumétrico particular para la morfología de los cristales formados.

Existen dos familias de métodos para obtener la densidad poblacional, la primera se basa en datos másicos, como los obtenidos en un juego de tamices, y la segunda se basa en conteos. La decisión de qué método utilizar se basa principalmente en los recursos y equipo disponible para el análisis. Para fines de esta investigación se utilizó un método de conteo microscópico con el cual se obtienen datos discretos de cuántos cristales se encuentran dentro de una categoría de tamaño determinada. Estos datos se convierten a número de cristales por unidad de volumen con la ecuación

$$
\Delta \mathrm{N}_{\mathrm{i}}=\frac{\text { Frecuencia }}{k_{v} \bar{L}_{i}^{3}}
$$

haciendo uso del factor de forma que para prismas hexagonales es

$$
\mathrm{k}_{\mathrm{v}}=\frac{2 \sqrt{3}}{(2 \times 3.2204)^{2}}
$$

Estos datos de distribución acumulativa se transforman a densidad poblacional dividiéndolos entre la magnitud del rango de tamaños con la ecuación

$$
\mathrm{n}_{\mathrm{i}}=\frac{\Delta N_{i}}{\Delta L_{i}}
$$

Si se grafica el tamaño medio de cristal contra el logaritmo de la densidad poblacional se obtiene una recta con pendiente $m$ e intercepto $\ln \left(n^{\circ}\right)$ a partir de la cual se obtienen las tasas de crecimiento

$$
\mathrm{G}=\frac{1}{m \tau}
$$

y de nucleación

$$
\mathrm{B}^{\mathrm{o}}=n^{o} G
$$

Es posible obtener también el número total de cristales por unidad de volumen con la ecuación

$$
\mathrm{N}_{\mathrm{T}}=n^{o} G \tau
$$

\section{Parte Experimental}

Para analizar la cristalización del óxido de zinc fue necesario encontrar un solvente adecuado. Se encontró que el $\mathrm{ZnO}$ no es soluble en agua ni en alcoholes, [5] pero es soluble en soluciones de cloruro de amonio [6]. Se encontraron datos de solubilidad a una concentración 
de cloruro de amonio fija, reportados a diferentes temperaturas. Para el experimento se seleccionaron $20^{\circ} \mathrm{C}$ y $40^{\circ} \mathrm{C}$ como las temperaturas baja y la alta, respectivamente; esto con el fin de sobresaturar la solución y lograr la cristalización del óxido de zinc.

\section{Cristalizador MSMPR}

Se realizaron 3 repeticiones del experimento con 3 variaciones de tiempo de residencia cada una, para dar un total de 9 corridas. El sistema utilizado se ensambló a manera de cristalizador continuo tipo MSMPR. Se muestra un esquema simplificado del ensamble en laFigura 2. Su finalidad principal fue llevar la solución saturada de una T1 a una T2, forzando así al óxido de zinc a cristalizar y formar una suspensión. El sistema consistió en dos tanques agitados, uno a $40^{\circ} \mathrm{C}$ en el que se disolvía continuamente el óxido de zinc sólido que entraba, y otro a menor temperatura, en el que ocurría la cristalización.

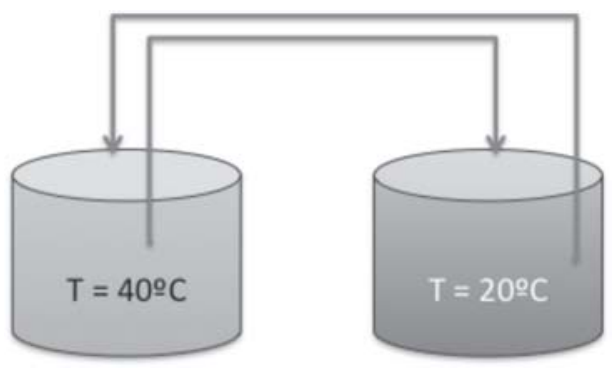

Figura 2. Diagrama simplificado del proceso.

Se preparó la solución de cloruro de amonio a una concentración de $23 \%$, y se disolvieron en ella $18.4 \mathrm{~g}$ de óxido de zinc. Esta solución se calentó a $40^{\circ} \mathrm{C}$ y se mantuvo sobre una plancha para mantener la temperatura y agitación constante. Por el otro lado, el tanque frío se mantuvo dentro de un baño refrigerado para mantener la temperatura a $20^{\circ} \mathrm{C}$. Una vez disuelto se inició cada corrida. En la Figura 3 se puede observar una foto del sistema real.

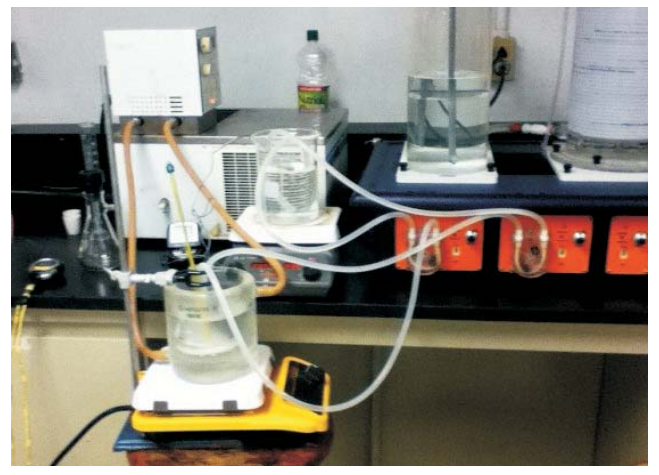

Figura 3. Cristalizador continuo de $\mathrm{ZnO}$

Cada tanque se conectó a una entrada y una salida de una bomba peristáltica, por medio de las cuales avanzaban los cristales de un tanque a otro de manera continua. Ambas bombas manejaron el mismo flujo. Después de cumplido el tiempo estimado para alcanzar el estado estable $t_{\text {est }}$ (calculado como 8 veces $\tau$ ) para cada corrida, se tomó una muestra de $100 \mathrm{ml}$ a la salida del y se filtró utilizando un matraz Kitasato y una bomba de succión. Se etiquetaron y almacenaron por separado la fase líquida y la fase sólida, esta última utilizando papel filtro. Este procedimiento se repitió para tres flujos diferentes como se muestra en la Tabla 2.

Tabla 2. Flujos para cada una de las corridas experimentales

\begin{tabular}{|c|c|c|c|}
\hline $\begin{array}{c}\text { Flujo } \\
{[\mathrm{ml} / \mathrm{min}]}\end{array}$ & $\begin{array}{c}\tau \\
{[\mathrm{min}]}\end{array}$ & $\begin{array}{c}\mathrm{t}_{\text {est }}(8 \mathrm{\tau}) \\
{[\mathrm{min}]}\end{array}$ & $\begin{array}{c}\text { V Cristalizador } \\
{[\mathrm{ml}]}\end{array}$ \\
\cline { 1 - 3 } 73.17 & 1.37 & 10.93 & \multirow{2}{*}{100} \\
\cline { 1 - 3 } 81.63 & 1.23 & 9.80 & \\
\hline 83.92 & 1.19 & 9.53 & \\
\hline
\end{tabular}

\section{Titulación}

Las muestras sólidas se dejaron secar para posterior análisis bajo el microscopio. La muestra de la fase líquida se tituló después de terminado el experimento, esto con el fin de obtener la concentración final alcanzado el estado estable. Se prepararon soluciones de $\mathrm{NaOH}$ y $\mathrm{HCl} 0.1 \mathrm{~N}$. Se preparó también un blanco para utilizar como referencia, combinando $6.5 \mathrm{ml}$ de una solución de cloruro de amonio puro, sin $\mathrm{ZnO}$ disuelto y $43.5 \mathrm{ml}$ de $\mathrm{HCl}$. Se llenó la bureta con $50 \mathrm{ml}$ de $\mathrm{NaOH}$. De la muestra líquida se tomaron $6.5 \mathrm{ml}$ y se mezclaron con $43.5 \mathrm{ml}$ de $\mathrm{HCl}$, para un total de $50 \mathrm{ml}$. Se agregaron 4 gotas de naranja de metilo como indicador y se tituló hasta el vire, de un color rosa a naranja claro. Esto se repitió para cada una de las muestras. Se tomó nota del volumen consumido de $\mathrm{NaOH}$ para realizar el cálculo de la concentración final utilizando la fórmula presentada por Sabura begum [7]

$$
\mathrm{M}_{\mathrm{ZnO}}=4.07(B-A)
$$

en la que $M_{Z n O}$ [g] es la masa de óxido de zinc presente en la muestra $B[\mathrm{ml}]$ es el volumen de la solución de $\mathrm{NaOH}$ consumida en el blanco y $A[\mathrm{ml}]$ es el volumen de la solución de $\mathrm{NaOH}$ consumido en la titulación de la muestra con $\mathrm{ZnO}$.

\section{Conteo Microscópico}

Las muestras sólidas se analizaron bajo un microscopio óptico, colocando una pequeña cantidad de la muestra en un portaobjetos, agregando un poco de etanol como solvente y observando con la lente de menor aumento para apreciar el mayor número posible de cristales. Posteriormente se analizaron con el software Microsoft PowerPoint, trazando líneas a lo largo de la longitud característica $L$ de cada cristal como se observa en la 
Figura 4 y registrando las dimensiones de dichas líneas para tres imágenes representativas por cada una de las nueve corridas.

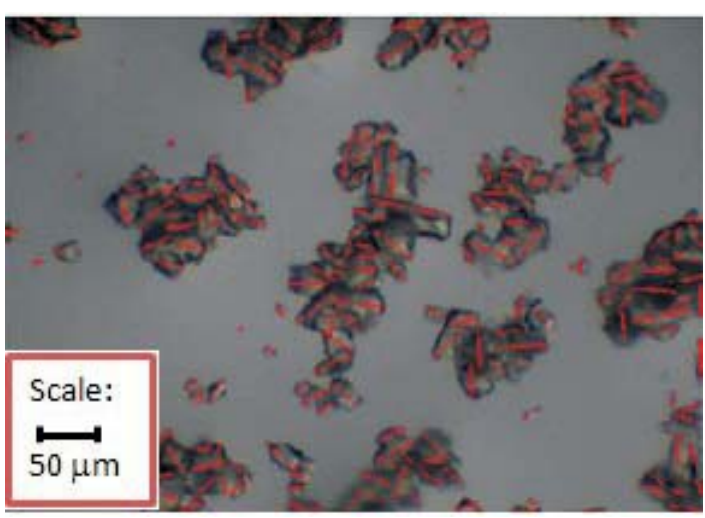

Figura 4. Determinación del tamaño de los cristales

\section{Resultados y discusión}

Para realizar el balance poblacional de cada una de las nueve corridas, primeramente se determinó el tamaño de los cristales a partir de una escala existente para el microscopio óptico. La longitud equivalente de la línea representante de $50 \mu \mathrm{m}$ es de $0.63 \mathrm{~cm}$, ver figura 5 .

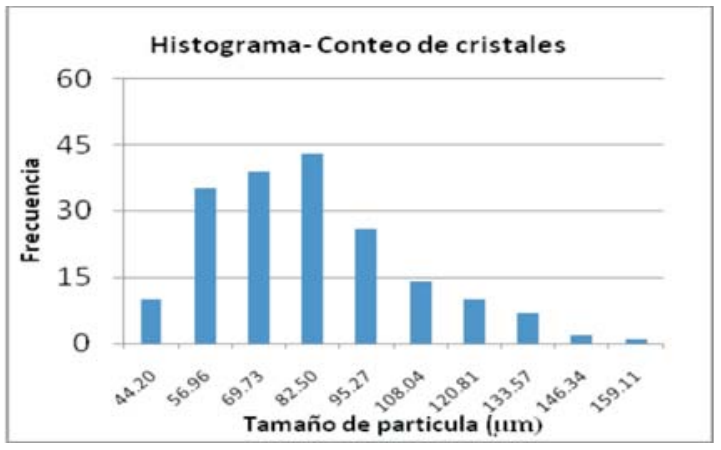

Figura 5. Histograma a partir de los datos de la corrida 2-a

Se obtuvieron 9 histogramas de los cuales se determinaron rangos de tamaño y la frecuencia de cristales pertenecientes a cada rango. A partir de estos datos se obtuvieron tamaños promedio $\bar{L}$ en cada rango y a partir de este dato y la frecuencia se obtuvo la función de número de cristales $\triangle N i$ utilizando la Ecuación 7. Al dividirlo entre el tamaño del rango $\Delta L_{i}$ se obtuvo la densidad poblacional con la Ecuación 9.

Para realizar el cálculo de la función de número de cristales se supuso que la forma de los cristales era de prisma hexagonal, con una relación promedio entre la longitud $L$ y su anchura $2 a$ de 1:3.22 por lo que se determinó un factor volumétrico de forma $k v$, con la fórmula

$$
\mathrm{k}_{\mathrm{v}}=2\left(\frac{a}{L}\right)^{2} \sqrt{3}=2\left(\frac{L / 2 X 3.22}{L}\right)^{2} \sqrt{3}
$$

resultando en una $k_{v}$ de 0.0835 .

Se graficó el tamaño promedio de cristal $\bar{L}$ contra el logaritmo de la densidad poblacional para cada rango $\ln \left(\right.$ ni) para obtener la pendiente $\mathrm{m}$ y el intercepto $\ln \left(n^{\circ}\right)$ de la función como en la Figura 6. A partir de estos valores se calculó la tasa de crecimiento $G$ con la ecuación 10 y la tasa de nucleación $B^{o}$ con la Ecuación 11. En el caso de la corrida 2-c, se obtuvo una pendiente de 0.0848 y un intercepto de 26.295. A continuación se muestra un ejemplo de una gráfica con los valores obtenidos a partir de los cuales se obtuvieron los valores de la Tabla 3.

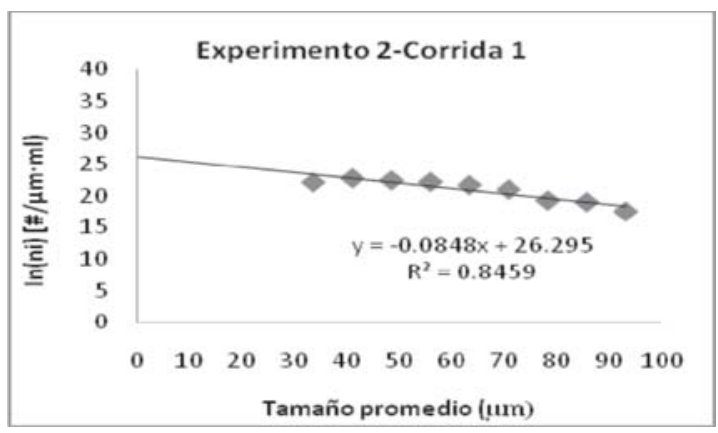

Figura 6. Tamaño medio vs logaritmo de la densidad poblacional

Para cada experimento se obtuvo una tabla similar a la siguiente:

Tabla 3. Valores de pendiente e intercepto de las corridas 2b,c

\begin{tabular}{|c|r|r|r|}
\hline Corrida & \multicolumn{1}{|c|}{$\tau$} & $\mathrm{m}$ & \multicolumn{1}{|c|}{$\ln \left(\mathrm{n}^{\circ}\right)$} \\
\hline 2-a & 0.1583 & 0.0585 & 25.092 \\
\hline 2-b & 0.181 & 0.0681 & 25.259 \\
\hline 2-c & 0.205 & 0.0848 & 26.295 \\
\hline
\end{tabular}

La obtención de los datos anteriores fue necesaria para llevar a cabo los cálculos de las tasas de crecimiento y nucleación con las ecuaciones 10 y 11, respectivamente. Así se generaron datos para las tasas de crecimiento y nucleación de cada corrida como se muestra en la Tabla 4. 
Tabla 4. Tasas de crecimiento y nucleación

\begin{tabular}{|l|l|l|}
\hline Corrida & $\mathrm{G}$ & $\mathrm{B}$ o \\
\hline $1-\mathrm{a}$ & 0.135 & $1.31 \mathrm{E}+05$ \\
$1-\mathrm{b}$ & 0.083 & $7.95 \mathrm{E}+05$ \\
$1-\mathrm{c}$ & 0.068 & $1.23 \mathrm{E}+06$ \\
$2-\mathrm{a}$ & 0.108 & $1.35 \mathrm{E}+06$ \\
$2-\mathrm{b}$ & 0.081 & $1.37 \mathrm{E}+06$ \\
$2-\mathrm{c}$ & 0.058 & $3.10 \mathrm{E}+06$ \\
$3-\mathrm{a}$ & 0.114 & $3.80 \mathrm{E}+05$ \\
$3-\mathrm{b}$ & 0.091 & $1.45 \mathrm{E}+06$ \\
$3-\mathrm{c}$ & 0.065 & $1.18 \mathrm{E}+06$ \\
\hline
\end{tabular}

Fue necesario establecer las concentraciones con las que se trabajó para determinar la fuerza impulsora con la que se generaron el crecimiento y la nucleación. En todas las corridas se trabajó con una concentración inicial de $23 \mathrm{~g} / \mathrm{L}$ y una concentración de saturación de $10 \mathrm{~g} / \mathrm{L}$. La concentración final para cada corrida se obtuvo a partir de las titulaciones, cuyos resultados se presentan a continuación:

Tabla 5.Concentraciones finales obtenidas mediante titulación

\begin{tabular}{|c|r|r|}
\hline Corrida & $\mathrm{NaOH}[\mathrm{ml}]$ & $\mathrm{C}[\mathrm{g} / \mathrm{L}]$ \\
\hline $1-\mathrm{a}$ & 19.1 & 18.8 \\
\hline $1-\mathrm{b}$ & 26.6 & 14.1 \\
\hline $1-\mathrm{c}$ & 28.6 & 12.8 \\
\hline $2-\mathrm{a}$ & 23.9 & 15.8 \\
\hline $2-\mathrm{b}$ & 28.4 & 14.0 \\
\hline $2-\mathrm{c}$ & 29.6 & 12.2 \\
\hline $3-\mathrm{a}$ & 22.4 & 16.7 \\
\hline $3-\mathrm{b}$ & 26.4 & 14.2 \\
\hline $3-\mathrm{c}$ & 29.2 & 12.5 \\
\hline
\end{tabular}

A partir de las concentraciones y las tasas de crecimiento y nucleación obtenidas para cada corrida, se prosiguió a calcular la constante de crecimiento $\mathrm{k}_{g}$, la constante de nucleación $\mathrm{k}_{b} \mathrm{y}$ los respectivos órdenes para las cinéticas $g$ y b a partir de una regresión lineal.

Los resultados obtenidos se resumen en la Tabla 6

Tabla 6.Contantes y órdenes para el crecimiento y la nucleación

\begin{tabular}{|lr|cr|}
\hline \multicolumn{2}{|c|}{ Nucleación } & \multicolumn{2}{|c|}{ Crecimiento } \\
\hline $\mathrm{b}$ & -1.5 & $\mathrm{~g}$ & 0.6 \\
\hline $\mathrm{k}_{\mathrm{b}}$ & $1.00 \mathrm{E}+06$ & $\mathrm{k}_{\mathrm{g}}$ & 0.0364 \\
\hline
\end{tabular}

La regresión de la tasa de crecimiento $G$ se puede ver en la Figura 7, la cual demuestra tener una $\mathrm{R}^{2}$ de 0.9893. Esto se considera bastante confiable. Asimismo, se determinó el porcentaje de error para verificar la exactitud de los valores obtenidos para las constantes $\mathrm{k}_{g}$ y $\mathrm{k}_{b}$, así como para los exponentes $g \mathrm{y}$ $b$. Para $\mathrm{k}_{g}$ y $g$ se obtuvo un porcentaje de error de $2.07 \%$ y para $\mathrm{k}_{b} \mathrm{y} b$ de $31.51 \%$.

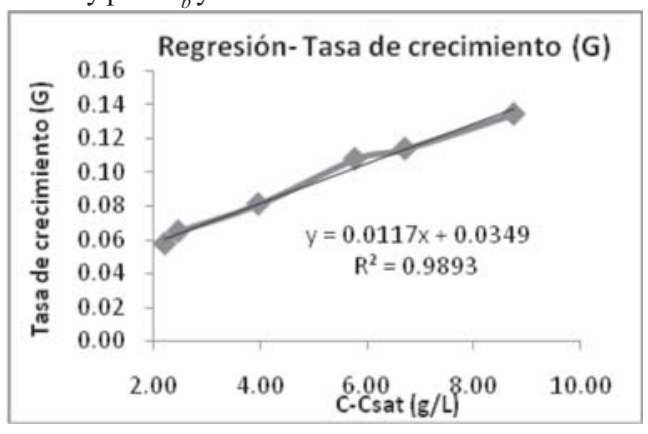

Figura 7. Regresión lineal para la tasa de crecimiento $(\mathrm{G})$

\section{Cálculos muestra}

Para determinar el volumen de los cristales se supuso que su forma era de prisma hexagonal, por lo que se

Tabla 7. Cálculo para determinación de constante $\mathrm{k}_{\mathrm{v}}$

\begin{tabular}{|c|l|}
\hline Ecuación & \multicolumn{1}{c|}{ Explicación } \\
\hline$\frac{2 \sqrt{3}}{\left(\text { Apotema }^{*} 2\right)^{2}}=\frac{2 \sqrt{3}}{(3.220401 * 2)^{2}}=1.083505$ & $\begin{array}{l}\text { Cálculo para la constante } \mathrm{k}_{\mathrm{v}} \text { para balance } \\
\text { poblacional }\end{array}$ \\
\hline
\end{tabular}

Los cálculos muestra para la determinación de cada uno de los parámetros necesarios para realizar el balance poblacional y obtener las tasas de crecimiento y nucleación se muestran a continuación: 
Los cálculos muestra para la determinación de cada uno de los parámetros necesarios para realizar el balance poblacional y obtener las tasas de crecimiento y nucleación se muestran a continuación:

Tabla 8. Cálculos muestra

\begin{tabular}{|c|c|}
\hline Ecuación & Explicación \\
\hline$\frac{0.6261 \mathrm{~cm}^{* 50 \mu m}}{0.63 \mathrm{~cm}}=49.69 \mu \mathrm{m}$ & $\begin{array}{l}\text { Cálculo para la determinación del tamaño } \\
\text { de los cristales a partir de la escala } \\
\text { existente }\end{array}$ \\
\hline$\frac{10 *\left(1 \times 10^{3}\right)}{0.083505^{*} 33.3245^{3}}=3.2707 \times 10^{10}$ & $\begin{array}{l}\text { Cálculo para la determinación de la función } \\
\text { del número de cristales }\left(\Delta \mathrm{N}_{\mathrm{i}}\right) \text { Factor de } \\
\text { conversión de unidades: } 1 \times 10^{3}\end{array}$ \\
\hline$\frac{3.2070 \times 10^{10}}{7.5}=4.2942 \times 10^{9}$ & $\begin{array}{l}\text { Cálculo para determinación del balance } \\
\text { poblacional }\left(\mathrm{n}_{\mathrm{i}}\right) \text {. }\end{array}$ \\
\hline$\left\lceil\frac{0.0848 \mu \mathrm{m}^{*} 0.1583 \mathrm{~h}}{1000 \mathrm{~mm}}\right]^{-}=0.0745 \mathrm{~mm} / \mathrm{h}$ & $\begin{array}{l}\text { Cálculo para la determinación de tasa de } \\
\text { crecimiento }(G)\end{array}$ \\
\hline$\frac{e^{26.295}}{1000 \mathrm{~mm}}=2.629 \times 10^{8} \# / \mathrm{mmL}$ & $\begin{array}{l}\text { Cálculo para la determinación de la } \\
\text { densidad poblacional del número de } \\
\text { cristales cuando el tamaño tiende a } 0\left(\mathrm{n}^{\circ}\right) \text {. }\end{array}$ \\
\hline $0.07448 \frac{m m}{h} \times 0.1583 h \times 2.629 \times 10^{8} \frac{\#}{m^{*} L}=3.1 \times 10^{6} \frac{\#}{L}$ & Cálculo para la determinación de $\mathrm{N}_{\mathrm{T}}$ \\
\hline $0.07448 \frac{\mathrm{mm}}{\mathrm{h}} \times 2.689 \times 10^{8} \frac{\#}{\mathrm{~mm}^{*} \mathrm{~L}}=.958 \times 10^{7} \frac{\#}{\mathrm{hL}}$ & $\begin{array}{l}\text { Cálculo para la determinación de la tasa de } \\
\text { nucleación. }\end{array}$ \\
\hline $\ln (G)=\ln (K)+g \ln \left(C-C_{\text {sat }}\right)$ & $\begin{array}{l}\text { Regresión lineal para cálculo de constantes } \\
\mathrm{k}_{\mathrm{g}} \mathrm{y} \mathrm{k}_{\mathrm{b}} \mathrm{y} \text { exponentes } \mathrm{g} \text { y b. }\end{array}$ \\
\hline
\end{tabular}

\section{Nomenclatura}

A volumen de $\mathrm{NaOH}$ consumido en titulación

B volumen de $\mathrm{NaOH}$ consumida en blanco

b orden de la cinética de nucleación

$\mathrm{B}^{\mathrm{o}} \quad$ tasa de nucleación

C concentración de una solución

g orden de la cinética de crecimiento

G tasa de crecimiento basada en una dimensión característica

$\mathrm{k}_{\mathrm{b}} \quad$ constante cinética de nucleación

$\mathrm{k}_{\mathrm{g}} \quad$ constante cinética de crecimiento

$\mathrm{k}_{\mathrm{v}} \quad$ factor volumétrico de forma

L longitud característica de partícula

M masa de óxido de zinc en la muestra
$\mathrm{M}_{\mathrm{T}} \quad$ masa total de partículas por unidad de volumen

$\mathrm{n}, \mathrm{n}(\mathrm{L})$ distribución de densidad de población

$\mathrm{N}, \mathrm{N}(\mathrm{L})$ distribución del número de partículas

$\mathrm{n}^{\mathrm{o}} \quad$ densidad de población de tamaño cero

$\mathrm{N}_{\mathrm{T}}$ número total de partículas por unidad de volumen

T temperatura

t tiempo

$\Delta \mathrm{N}_{\mathrm{i}} \quad$ cristales dentro del rango por unidad de volumen

$\rho \quad$ densidad de partícula

$\tau \quad$ tiempo de residencia

De acuerdo con los resultados obtenidos, se puede ver que conforme aumenta la sobresaturación, es decir C$\mathrm{C}_{\text {sat, }}$, aumenta la tasa de crecimiento $G$, la cual, al ser graficada contra la sobresaturación, resulta ser aproximadamente una línea recta y coincide con el orden 
de crecimiento $g$ el cual fue de 0.6. La constante de crecimiento $k_{g}$ obtenida fue de 0.036 , que resulta un valor coherente, arrojando un porcentaje de error para la función de crecimiento de $2.07 \%$. La constante de nucleación $k_{b}$ fue de $1 \times 10^{6}$ y el orden de la cinética $b$ fue de -1.5 .

Se puede ver que el aumento de la tasa de crecimiento depende de la sobresaturación $\mathrm{y}$, por otro lado, a una mayor sobresaturación se obtiene una tasa de nucleación $B^{o}$ menor, de acuerdo con los resultados anteriores.

Se puede decir que los cristales contados estuvieron en crecimiento, y es por esto que los datos y resultados son más precisos para crecimiento y no para nucleación. No se pudieron observar cristales pequeños ya que no se tenía una forma adecuada o la potencia del microscopio necesarias para medir cristales de esa magnitud.

Los resultados de nucleación no pudieron ser cuantificados de manera exacta y esto concuerda con lo encontrado en la literatura ya que ésta menciona que se presentan diversos obstáculos para su determinación [4]. Si se quisiera favorecer la nucleación se tendría que sobresaturar rápidamente $\mathrm{y}$ hacer un cambio de temperatura muy drástico.

Los resultados están más inclinados a representar la tasa de crecimiento que los de nucleación debido a las limitantes presentadas. El error para nucleación fue $31.51 \%$ comparado con $2.07 \%$ para el crecimiento, como se mencionó anteriormente. Esto se puede deber a posibles errores que se presentaron durante la etapa de experimentación, tales como: temperaturas no constantes debido a diversos factores como flujo volumétrico, tiempos, etc., la forma en la que se contaron los cristales debido a que el método para contarlos fue muy impreciso ya que se hizo manualmente y por personas diferentes para cada corrida y a la capacidad del microscopio óptico debido a que no se alcanza a ver cristales más pequeños por lo que éstos no pudieron ser medidos.

\section{Conclusiones}

A partir de la presente investigación, es claro que los parámetros obtenidos para describir las fases de la cristalización del óxido de zinc: constante de crecimiento de 0.0364 , constante de nucleación de $1 . \times 10^{6}$, así como los órdenes de reacción de cada fenómeno, 0.6 y -1.5, respectivamente; ayudan a comprender la relación entre la sobresaturación de una solución y las tasas de crecimiento y nucleación. Como se observó, la relación tiene una tendencia lineal para el fenómeno de crecimiento. Se observaron resultados concluyentes en esta fase. Sin embargo, como se mencionó anteriormente, la parte de nucleación del modelo está mucho más limitada, y se recomendaría utilizar el mismo, si acaso, con mucho cuidado. Quizás sea aún más deseable repetir los experimentos y profundizar más en este fenómeno, pues no se concibe aún una tendencia clara para éste.
Como recomendaciones para un futuro, si se repitieran los experimentos, lo ideal sería monitorear bien las temperaturas de operación y asegurarse de que siempre haya un mezclado lo más homogéneo posible en los tanques. Para la observación de partículas en el microscopio, se podrían obtener mejores resultados con un microscopio más potente, pues se podrían apreciar las partículas más pequeñas o justo en la fase de nucleación. Respecto al método de conteo, lo ideal sería utilizar un método de conteo estandarizado y que una misma persona realice los conteos, pues el proceso varía mucho de una persona a otra.

\section{Referencias}

1. K. A. Berglund, «Analysis and measurement of crystallization utilizing the population balance».En Handbook of industrial crystallization, A. S. Myerson, Ed., Boston: Butterworth-Heinemann, 1993.

2. F. Grases, A. Costa y O. Söhnel, Cristalización en disolución: Conceptos básicos, Barcelona: Reverté, 2000.

3. A. Mersmann, Ed., Crystallization Technology Handbook, $2^{\mathrm{a}}$ ed., Nueva York: Marcel Dekker, 2001.

4. D. Skoog y D. West, Introducción a la química analítica, Sevilla: Reverté, 2002.

5. R. J. Lewis Sr., Hawle'ys condensed chemical dictionary, $15^{\mathrm{a}}$ ed. ed., Wiley, 2007.

6. W. Burrows, Zinc oxide recovery process Patent 3849121, Atlanta, GA, 1974.

7. P. Sabura begum, M. Mohammed Yusuff y J. Rani, «Preparation and characterization of zinc oxide,» de Studies on the use of nano zinc oxide and modified silica in NR, $C R$, and SBR, Kochi, Cochin University of Science and Technology, 2009, pp. 61-74.

8. A. C. Dodd y otros, «Effect of particle size on the photocatalytic activity of nanoparticulate zinc oxide.,» Journal of Nanoparticle Research, 2006, vol. 8, $\mathrm{n}^{\circ}$ 1, pp. 4351

9. E. Greene y otros, «Low-Temperature Wafer-Scale Production of $\mathrm{ZnO}$ Nanowire Arrays.,» Weinheim, WileyVCH Verlag GmbH \& Co, 2003, pp. 3031-3034.

10. H. Morkoç y Ü. Özgür, «General Properties of $\mathrm{ZnO,»} \mathrm{de}$ Zinc Oxide: Fundamentals, Materials and Device Technology, Weinheim, Wiley-VCH Verlag GmbH \& Co., 2009, p. 2.

11. A. S. Myerson, Handbook of industrial crystallization, A. S. Myerson, Ed., Boston: Butterworth-Heinemann, 1993.

12. M. Sadat Mohajerani, A. Lak y A. Simchi, «Effect of morphology on the solar photocatalytic behavior of $\mathrm{ZnO}$ nanostructures,» Journal of Alloys and Compounds, 2009, vol. $485, \mathrm{n}^{\circ} 1-2$, pp. 616-620.

13. A. Taubert, G. Glasser y D. Palms, «Kinetics and Particle Formation Mechanism of Zinc Oxide Particles in PolymerControlled Precipitation from Aqueous Solution,» Langmuir, 2002, vol. 18, $\mathrm{n}^{\circ} 11$, p. 4488-4494.

14. Y. Peng, A.-W. Xu, B. Deng, M. Antoniett y H. Cölfen, «Polymer-Controlled Crystallization of Zinc Oxide Hexagonal Nanorings and Disks,» The Journal of Physical Chemistry B, 2006, vol. 110, $\mathrm{n}^{\circ}$ 7, pp. 2988-2998. 
15. H. Wanga, J. Xiea, K. Yanc y M. Duana, «Growth Mechanism of Different Morphologies of $\mathrm{ZnO}$ Crystals Prepared by Hydrothermal Method,» Journal of Materials Science \& Technology, 2011, vol. 27, $\mathrm{n}^{\circ}$ 2, p. 158.

16. S. Kalpakjian y S. Schmid, Manufactura, Ingeniería y tecnología, México: Pearson Education, 2002.
17. D. Pasto y C. Johnson, Determinación de estructuras orgánicas, Barcelona: Reverté, 2003. 